\title{
Resource development in otolaryngology-head and neck surgery: an analysis on patient education resource development
}

\author{
Jeremy Goldfarb ${ }^{1 \dagger}$, Vishaal Gupta ${ }^{1 \dagger}$, Heather Sampson $^{2}$ and Albino Chiodo ${ }^{3^{*}}$
}

\begin{abstract}
Background: There is a need for educational tools in the consenting process of otolaryngology-head and neck procedures. A development strategy for the creation of educational tools in otolaryngology-head and neck surgery, particularly pamphlets on the peri-operative period in an adenotonsillectomy, is described.

Methods: A participatory design approach, which engages key stakeholders in the development of an educational tool, is used. Pamphlets were created through a review of traditional and grey literature and then reviewed by a community expert in the field. The pamphlets were then reviewed by an interdisciplinary team including educational experts, and finally by less vulnerable members of the target population. Questionnaires evaluating the pamphlets' content, layout, style, and general qualitative features were included.

Results: The pamphlets yielded high ratings across all domains regardless of patient population. General feedback was provided by a non-vulnerable patient population and final pamphlets were drafted.

Conclusions: By using a participatory design model, the pamphlets are written at an appropriate educational level to incorporate a broad audience. Furthermore, this methodology can be used in future resource development of educational tools.
\end{abstract}

Keywords: Patient education, Resource development, Otolaryngology-head and neck surgery, Surgical complications, Post-operative period

\section{Introduction}

Currently 19 out of 10,000 Canadian children receive an adenotonsillectomy [1]. It is one of the most common procedures performed by otolaryngologists-head and neck surgeons, and is also one of the most common operations in the pediatric population. Although the procedure is so widely performed, the intra-operative and post-operative periods can be quite stressful, and caring for a child undergoing surgery can be very challenging for guardians, necessitating proper supports.

During the intra-operative and post-operative periods, it is particularly important that caregivers are provided with effective education. Previous studies have looked into the

\footnotetext{
*Correspondence: achiodo@rogers.com

${ }^{\dagger}$ Equal contributors

${ }^{3}$ Department of Otolaryngology - Head and Neck Surgery, University of Toronto, Toronto East General Hospital, 825 Coxwell Avenue, Toronto, Ontario M4C 3E7, Canada

Full list of author information is available at the end of the article
}

informed consent process, and the effect verbal and written consenting tools have on patient knowledge of these periods. Aremuu et al. [2] demonstrated that the addition of a handout significantly altered recall of potential complications in otolaryngology-head and neck surgeries. Furthermore, Le et al. [3] found that although most parents were satisfied with the preoperative counseling, $94 \%$ of patients felt that a postoperative phone call the day after surgery was helpful; despite counseling, they hadn't realized how severe the throat pain would be. Kuo et al. [4] demonstrated that patients lack awareness of symptoms they might expect post-adenotonsillectomy. Throat pain, bleeding, and voice changes are all common during the postoperative period, and their triage requires an informed caregiver equipped with appropriate educational tools.

Even though there is an increasing need for educational tools in otolaryngology, the most effective medium for peri-operateive surgical education in adenotonsillectomy 
is still debated. Adams et al. [5] found no significant difference in knowledge retention between verbal counseling, counseling and a written handout, and counseling and a video, and also concluded that the otolaryngologist-head and neck surgeon remained the most important source of information. This study, however, was performed on well-educated caregivers and did not provide a pamphlet development procedure. A primer for surgical pamphlet development is currently missing in the literature.

The aim of this study is to provide an approach for developing educational tools in otolaryngology-head and neck surgery that will be effective across multiple populations.

\section{Methods}

A mixed methods approach was utilized and adhered very closely to the methodology described by Adirim et al. [6] for developing and evaluating an educational pamphlet. Two pamphlets were drafted-an pre-operative pamphlet outlining complications and a post-operative pamphletdescribing the postoperative course or care of the patient.

\section{Development}

The development portion was conducted using a participatory design approach, engaging key stakeholders [7].

\section{Phase 1 - Pamphlet development and design}

The pamphlets were created using a systematic review of the current informational landscape in adenotonsillectomy peri-operative care. A review of traditional literature was done and pertinent information was included in the pamphlets. Multiple search strategies in Ovid MEDLINE were utilized and four review papers were selective as information sources [8-10]. Additionally, an environmental scan including online grey literature and similar pamphlets available at other Canadian otolaryngology-head and neck surgery clinics was done. Pamphlets created by McGill University [11] and the University of Mississippi [12] were included in the review process. Grey literature was reviewed using popular search mediums available to patients. Utilizing multiple search strategies, google.com, yahoo.com, and bing.com were searched. Websites providing lay information on adenotonsillectomy were reviewed. The pamphlets were written to be inclusive of individuals with minimal educational background as per the recommendations of McAllister et al. [13]. All the information was reviewed to create draft pamphlets which were audited by an experienced community otolaryngologist-head and neck surgeon before proceeding to Phase 2.

Phase 2 - Critical evaluation by diverse healthcare professionals The draft pamphlets from Phase 1 were distributed to a team of healthcare professionals for critical evaluation of content and style. The pamphlets were given to two adenotonsillectomy-performing otolaryngologists-head and neck surgeons, three nurses, one speech language pathologist, two educational experts, and one plain text editor. The pamphlets were edited based on the qualitative feedback from this group. (see "Key Stakeholders")

\author{
Key stakeholders \\ Otolaryngologist - head and neck surgeon \\ Nurses in Otolaryngology \\ Speech Language Pathologist \\ Educational Experts \\ Plain Text Editors \\ Care-givers of Non-vulnerable Patients \\ Fellow Medical Student
}

\section{Phase 3 - Evaluation by less vulnerable members of the target population}

This phase involved evaluation of style and content by guardians of children who have undergone adenotonsillectomy, and whose children have successfully recovered from the surgery. Guardians were contacted after the follow-up visit, once an otolaryngologist-head and neck surgeon ensured there were no post-operative complications. They were asked to read the pamphlets and fill out a brief questionnaire to elicit qualitative feedback (see 'Patient Questionnaires' subsection). The pamphlets were again modified based on the target audience's evaluation, and final pamphlets were drafted. The final pamphlets were then assessed for readability using the Flesch-Kincaid readability test.

\section{Patient questionnaire}

Adenotonsillectomy Qualitative Feedback Questionnaire:

Thank you for your agreeing to participate on our resource development project. We very much appreciate your time and valuable feedback.

Name:

Relation to patient:

Surgical Complications Pamphlet:

Please provide feedback on the content of the pamphlet. (Was it too difficult? Is it appropriate for the target population? Should anything be removed? Should anything be added?)

Please provide feedback on the style of the pamphlet (Was the syntax appropriate? Was the language confusing?) Please provide feedback on the general layout of the pamphlet (Were the sections appropriately titled? Were the colours acceptable? Were the visual images appropriate?) General Feedback:

Post-Operative CarePamphlet:

Please provide feedback on the content of the pamphlet. (Was it too difficult? Is it appropriate for 


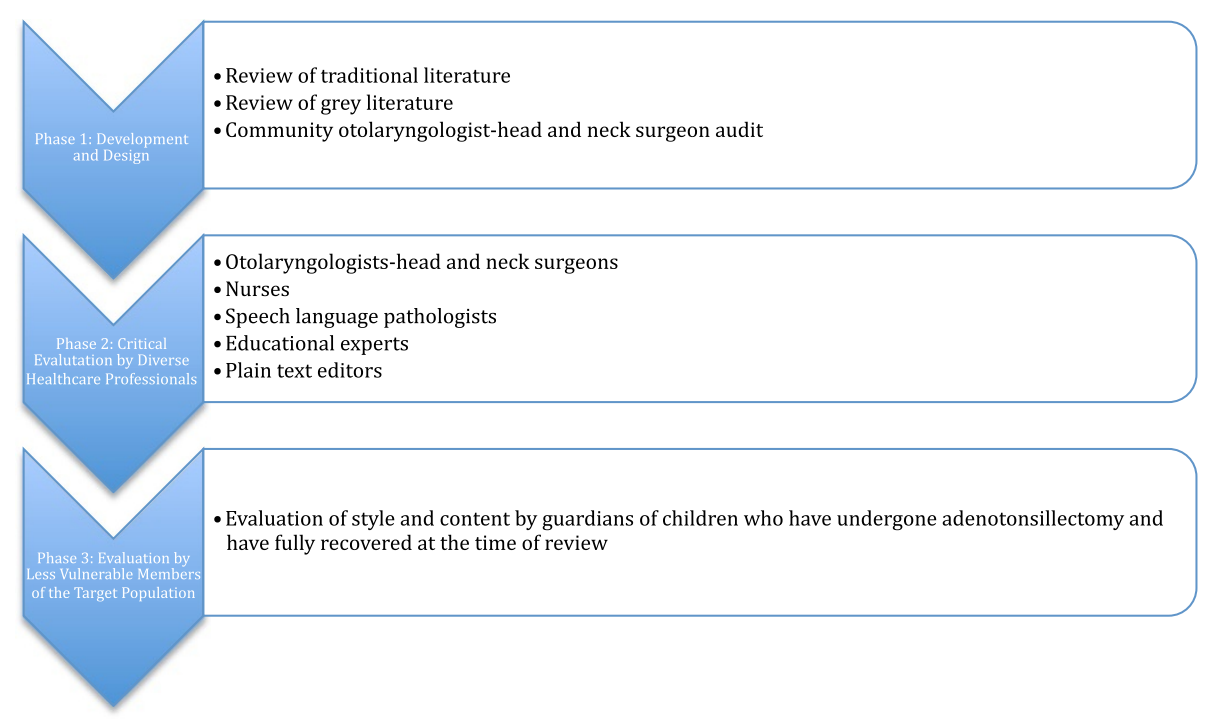

Figure 1 Development and evaluation workflow.

the target population? Should anything be removed? Should anything be added?)

Please provide feedback on the style of the pamphlet (Was the syntax appropriate? Was the language confusing?) Please provide feedback on the general layout of the pamphlet (Were the sections appropriately titled? Were the colours acceptable? Were the visual images appropriate?) General Feedback:

Figure 1 Development and evaluation process.

\section{Results}

The pamphlets were developed using the methods outlined. A multidisciplinary team was engaged that provided feedback on content, style, appropriateness of language, and overall effectiveness. The pamphlets were edited five times in an iterative process based on the feedback provided. Educational experts provided feedback on language difficulty and recommended simplification. Other themes that emerged in the multidisciplinary review were improving content layout and providing additional information on appropriate patient triage. These changes were made and incorporated into the draft given to non-vulnerable patients.

Ten non-vulnerable patients were then approached to participate in Part 1, Phase 3 of the study. Ten patientguardians completed the questionnaire during their intra-operative and post-operative visit. Nine of the ten participants had positive or no feedback on content, style, or layout. (Table 1) These changes were made and final pamphlets (Figures 2 and 3) were designed. The final pamphlets were also scored on the Flesch-Kincaid readability test. The Surgical Complications pamphlet scored at a Grade 11.5 level and the Post-Operative Complications pamphlet at a Grade 8.3 level.

\section{Discussion}

Educational materials are of limited value if patients cannot understand their content [4]; therefore, this resource development study aimed to create and evaluate a perioperative pamphlet for caregivers of children undergoing adenotonsillectomy to ensure it can be understood and effective for patients of all backgrounds.

Development of an effective resource depends on creating a resource written at an appropriate educational level [13]. A participatory design model, engaging key stakeholders in the design process, can be used to develop an appropriate resource [6]. Once an appropriate resource has been developed, it was shown by Aremu et. al. [2] that handouts improve recall in otolaryngology-head and neck procedures (62\% vs $51 \%$ ). Knowing this, a participatory design model should be employed to create peri-operative pamphlets for paediatric caregivers of various backgrounds.

Due to the time intensive nature of our participatory design model, engaging a large sample population was not

Table 1 Feedback from care-givers of non-vulnerable patients

\begin{tabular}{ll}
\hline Domain: & Feedback (Quotes): \\
\hline General: & "Additional online resources should be provided" \\
& "How much bleeding requires as emergency room visit?" \\
$\begin{array}{ll}\text { Content, Style, } \\
\text { and Layout: }\end{array}$ & "Very easy to follow" \\
& "Reflected my discussion with the doctor well" \\
& "Layout was a bit confusing" \\
& "Some language was too complicated" \\
\hline
\end{tabular}




\section{What are adenoids \& \\ tonsils?}

Tonsils are small round clumps of tissue located at the back of the throat. They are mostly made up of lymphoid tissue, and they help fight infections. Removal of tonsils will not affect a child's immune system and ability to fight infection. Adenoids are tonsil tissue located at the back of the nose.

Tonsillitis: an inflammation of the tonsils, usually caused by a viral/bacterial infection

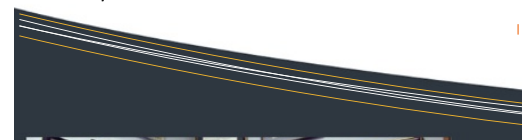

\section{ADENOTONSILLECTOMY}

What it is and what to be concerned about

A Patient Information Brochure

Brought to you by the:

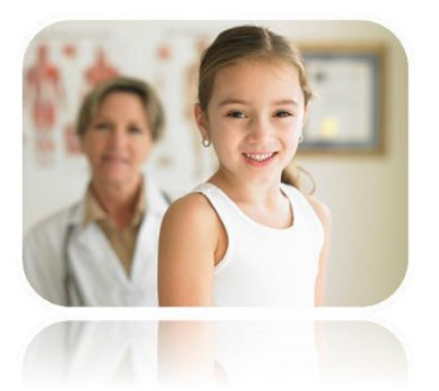

The Department of Otolaryngology-Head and

Neck Surgery,

Toronto East General Hospital

University of Toronto

Dr. A. Chiodo

416-510-9962

Dr. W. El Masri

416-406-6540

416-406-6540

Dr. O. Smith

416-465-0919

\section{What to do pre-operatively?}

Why do I need a adenotonsillectomy?

Reasons for an adenotonsillectomy include:

- Persistent, recurrent, or severe tonsillitis or strep infections;

- Large or swollen tonsils that make it hard to breathe or swallow;

- Sleep difficulty that may affect a child's daily activities;

- Snoring and obstructive sleep apnea;

- Abscess on the tonsils.

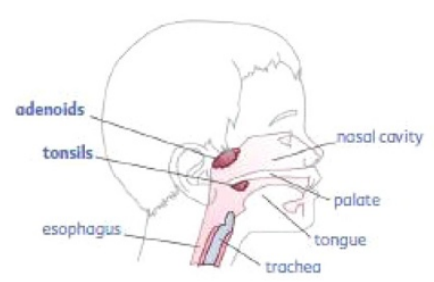

Aspirin and NSAID's (i.e. Tylenol) should be stopped;

- Ensure child understands the surgery and provide support.

\section{The Surgery}

General anaesthesia is used;

The surgery will take 30-45 minutes;

A complete tonsil removal is usually performed;

The tonsils are accessible through the mouth, no incision is required;

Your child will wake up in the recovery room and visitation is usually permitted;

Most children are allowed to go home the same day, but your child may have to stay overnight.

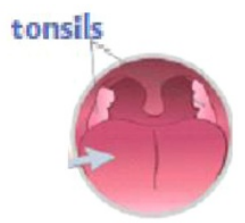

\section{What are the surgical} complications?

No surgery is without risks, but serious complications are rare.

\footnotetext{
General surgical risks, including anaesthesia;

- Bleeding (1\%-2\%) upto 48 hours;

Pain;

Infection;

Dehydration;

Failure to correct underlying condition;

Tooth chipping, lip burn,

temporamandibular joint (jaw) pain;

Voice changes;

Unintelligible speech;

Abnormal bleeding 2\%-4\% of cases;

Death $1 / 15000$ due to bleeding, airway obstruction, or anaesthesia.
}

Figure 2 Surgical complications pamphlet. 


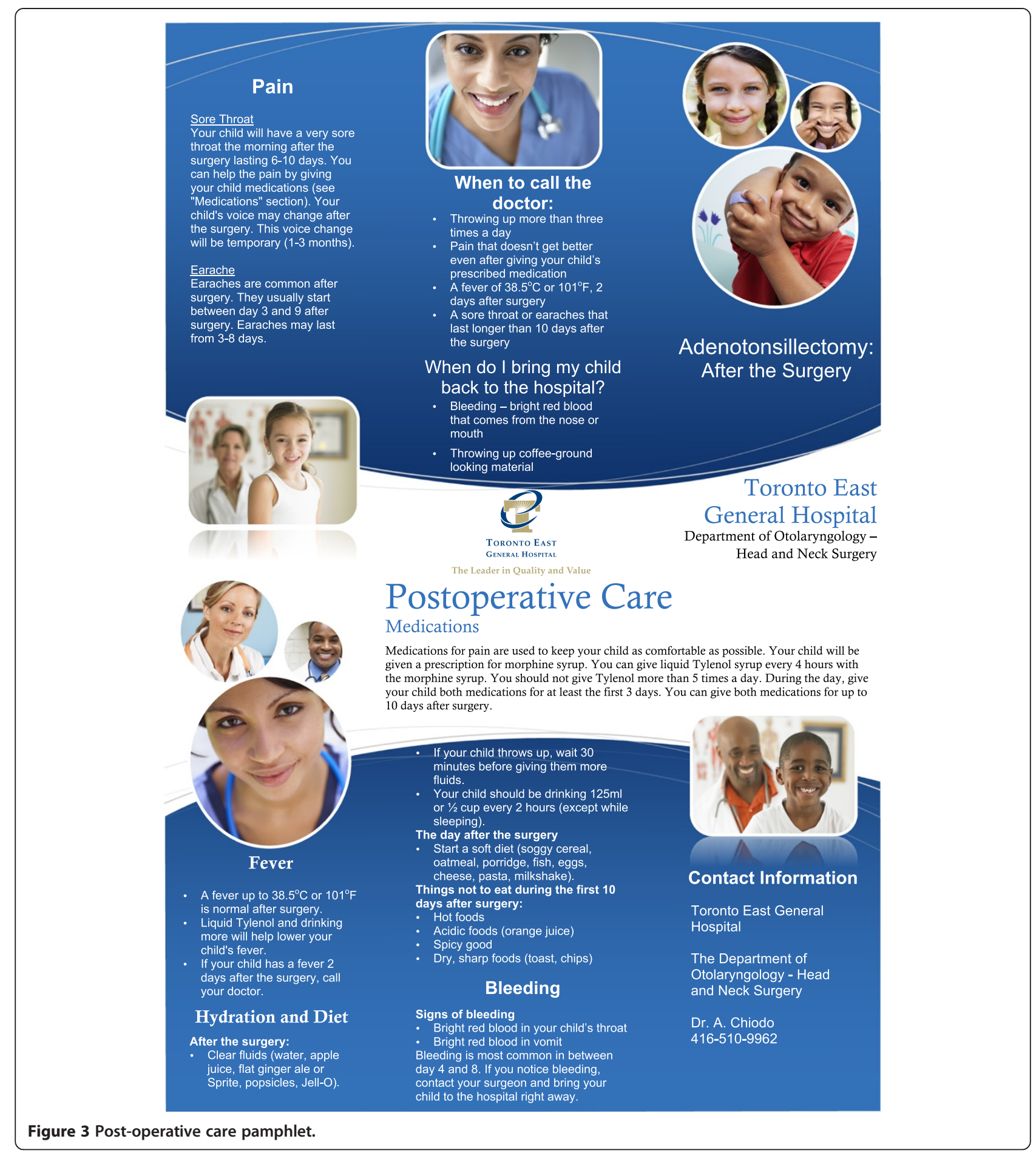

possible Therefore, the sample may not have been representative of the breadth of adenotonsillectomy patients, including non-English speakers and patients with poor literacy. Also, while the non-vulnerable patient caregivers positively reviewed the pamphlets, a more quantitative methodology assessing demographic information would be required to ensure the pamphlets are effective across socioeconomic strata. Ideally, the pamphlets would be reviewed by those with dyslexia, English as a second language, the visually impaired, and those with poor health literacy skills, to name a few, Once analyzed by these groups a more broadly inclusive pamphlet would be finalized. 


\section{Conclusions}

The aim of this study was to present an approach for development of future educational tools in otolaryngologyhead and neck surgery. It is important for physicians, as advocates, to provide up-to-date and understandable educational tools for patients. The above methodology has shown to be effective for creating a preliminary educational tool. It emphasizes interdisciplinary collaboration as well as the inclusion of end-users in the developmental process. However, further studies need to be conducted to determine the efficacy of such tools in various patient populations.

\section{Recommendations and considerations}

Utilizing the on-line format of this journal we would like to engage our colleagues in investigating the utility and value of this tool via the web based access (http://www. journalotohns.com/). We welcome readers/clinicians to download the brochure and questionnaires to use in their clinical environments. We look forward to receiving reader experience and evaluation of the patient education tool: we would be pleased to share recommended changes and edits to the brochure.

\section{Consent}

Written informed consent was obtained from the patient's guardian/parent/next of kin for the publication of this report and any accompanying images. Every effort was made to use stock images in the brochures.

\section{Competing interests}

The authors of this article have no competing interests to disclose and no conflicts of interest.

\section{Authors' contribution \\ VG and JG participated in the design of the study and drafted the manuscript. AC conceived of the study, carried out the study, and helped draft the manuscript. HS helped with study design, with data collection, and with drafting the manuscript. All authors read and approved the final manuscript. \\ Author details \\ ${ }^{1}$ Undergraduate Medical Education, Faculty of Medicine, University of Toronto, Toronto, Canada. ${ }^{2}$ Department of Family and Community Medicine, University of Toronto, Office of Research, Toronto East General Hospital, 825 Coxwell Avenue, Toronto, Ontario M4C 3E7, Canada. ${ }^{3}$ Department of Otolaryngology - Head and Neck Surgery, University of Toronto, Toronto East General Hospital, 825 Coxwell Avenue, Toronto, Ontario M4C 3E7, Canada.}

Received: 14 October 2013 Accepted: 30 June 2014

Published: 16 July 2014

\section{References}

1. Van Den Akker EH, Hoes AW, Burton MJ, Schilder AG: Large international differences in (adeno)tonsillectomy rates. Clin Otolaryngol Allied Sci 2004, 29(2):161-164.

2. Aremu SK, Alabi BS, Segun-Busari S: The role of informed consent in risks recall in otorhinolaryngology surgeries: verbal (nonintervention) vs written (intervention) summaries of risks. Am J Otolaryngol 2011, 32(6):485-489.
3. Le T, Drolet J, Parayno E, Rosmus C, Castiglione S: Follow-up phone calls after pediatric ambulatory surgery for tonsillectomy: what can we learn from families? J Perianesth Nurs 2007, 22(4):256-264.

4. Kuo M, Hegarty D, Johnson A, Stevenson S: Early post-tonsillectomy morbidity following hospital discharge: do patients and GPs know what to expect? Health Trends 1995, 27(3):98-100.

5. Adams MT, Chen B, Makowski R, Bevans S, Boseley M: Multimedia approach to preoperative adenotonsillectomy counseling. Otolaryngol Head Neck Surg 2012, 146(3):461-466.

6. Adirim T, Chafranskaia A, Nyhof-Young J: Investigating the impact of socioeconomic status on the effectiveness of a pamphlet on achieving and maintaining bone health in breast cancer survivors: a patient education resource development primer. J Cancer Educ 2012, 27(1):54-58.

7. Schuler D, Namioka A: Participatory Design: Principles and Practices. Hillsdale, New Jersey: L. Erlbaum Associates Inc; 1993.

8. Derkay CS, Darrow DH, LeFebvre SM: Pediatric tonsillectomy and adenoidectomy procedures. AORN J 1995, 62(6):887-904. quiz 906-810.

9. Ramos SD, Mukerji S, Pine HS: Tonsillectomy and adenoidectomy. Pediatr Clin N Am 2013, 60(4):793-807.

10. Statham MM, Myer CM 3rd: Complications of adenotonsillectomy. Curr Opin Otolaryngol Head Neck Surg 2010, 18(6):539-543.

11. Le T, Drolet J, Parayno E: Tonsillectomy and/or Adenoidectomy. In Montreal's Children Hospital. Montreal: McGill University Health Centre; 2014.

12. University Ear, Nose \& Throat, Speech and Hearing Clinic: Home Care After Tonsillectomy and Adenoidectomy. In Blair E Batson Hospital for Children. Mississippi: University of Mississippi Health Care; 2014.

13. McAllister KA, Clement WA: Readability and content of postoperative tonsillectomy instructions given to patients in Scotland, a completed audit cycle. Scott Med J 2012, 57(1):4-7.

doi:10.1186/s40463-014-0027-5

Cite this article as: Goldfarb et al:: Resource development in otolaryngology-head and neck surgery: an analysis on patient education resource development. Journal of Otolaryngology - Head and Neck Surgery 2014 43:27.

\section{Submit your next manuscript to BioMed Central and take full advantage of:}

- Convenient online submission

- Thorough peer review

- No space constraints or color figure charges

- Immediate publication on acceptance

- Inclusion in PubMed, CAS, Scopus and Google Scholar

- Research which is freely available for redistribution 